\title{
CD147 induces UPR to inhibit apoptosis and chemosensitivity by increasing the transcription of Bip in hepatocellular carcinoma
}

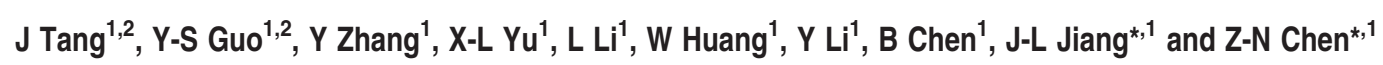

The unfolded protein response (UPR) is generally activated in solid tumors and results in tumor cell anti-apoptosis and drug resistance. However, tumor-specific UPR transducers are largely unknown. In the present study, we identified CD147, a cancer biomarker, as an UPR inducer in hepatocellular carcinoma (HCC). The expression of the major UPR target, Bip, was found to be positively associated with CD147 in human hepatoma tissues. By phosphorylating FAK and Src, CD147-enhanced TFII-I tyrosine phosphorylation at Tyr248. CD147 also induced p-TFII-I nuclear localization and binding to the Bip promoter where endoplasmic reticulum (ER) stress response element 1 (ERSE1) $(-82 /-50)$ is the most efficient target of the three ERSEs, thus increasing transcription of Bip. Furthermore, by inducing UPR, CD147 inhibited HCC cell apoptosis and decreased cell Adriamycin chemosensitivity, thus decreasing the survival rate of hepatoma-bearing nude mice. Together, these results reveal pivotal roles for CD147 in modulating the UPR in HCC and raise the possibility that CD147 is a target that promotes HCC cell apoptosis and increases the sensitivity of tumors to anti-cancer drugs. Therefore, CD147 inhibition provides an opportunity to enhance the efficacy of existing agents and represents a novel target for HCC treatment.

Cell Death and Differentiation (2012) 19, 1779-1790; doi:10.1038/cdd.2012.60; published online 18 May 2012

The uncontrolled growth and insufficient vascularization of a tumor mass lead to a stressed state in the tumor microenvironment, which includes low oxygen supply, nutrient deprivation and $\mathrm{pH}$ changes. Microenvironment stresses directly impinge on the luminal milieu of the endoplasmic reticulum (ER) and might be sufficiently severe to cause ER stress (ERS). ${ }^{1}$ These ERS conditions activate a range of cellular stress response pathways, including the unfolded protein response (UPR). ${ }^{2,3}$ UPR activation has profound consequences for tumor growth and resistance to radiotherapy and chemotherapy. ${ }^{4-6}$ Therefore, the UPR might be responsible for the failure of some patients to respond to treatment and is associated with a poor prognosis. ${ }^{7}$ Given the observation that UPR is correlated with certain types of aggressive tumors, drug resistance, recurrence and poor survival, ${ }^{8-11}$ the inhibition of the UPR pathway may yield more efficient treatments for cancer.

Although there is sufficient evidence to indicate that the UPR is generally activated in solid tumors, the UPR remains poorly characterized in cancers. The three major UPR transducers are inositol-requiring enzyme 1 (IRE1), pancreatic kinase-like ER kinase (PERK) and the UPR-specific transcription factor, activating transcription factor 6 (ATF6).
These factors all sense the presence of unfolded proteins in the ER lumen and transduce signals to the nucleus that activate the transcription of UPR target genes, such as Bip (also called glucose-regulated protein 78$)^{12-14}$ The overexpression of Bip as a result of the three major transducers has been reported in malignant breast disease, colon cancer and gastric and esophageal adenocarcinomas. ${ }^{15-18}$ It has also been reported that Bip overexpression is driven by a c-myb mutation in colon cancer. ${ }^{19}$ These results confirmed that the complexity of UPR has increased from the three major UPR transducers that are involved in simple pathways to multimolecules, which are involved in a multibranching signal transduction cascade. Tumor-specific UPR transducers should be explored to determine their usefulness as potential anti-cancer agents.

As a cancer biomarker, CD147 is highly expressed in hepatocellular carcinoma (HCC) but weakly expressed in normal liver tissues. ${ }^{20}$ Prior studies have demonstrated that CD147 plays important roles in the progression of HCC, including migration, proliferation, tumor recurrence and poor prognosis. ${ }^{20,21}$ Our recent study indicates that CD147 activates the FAK-PI3K-calcium $\left(\mathrm{Ca}^{2+}\right)$ signaling pathway by interacting with $\alpha 3 \beta 1$ integrin and disrupts the

\footnotetext{
${ }^{1}$ Department of Cell Biology, Cell Engineering Research Centre, State Key Laboratory of Cancer Biology, State Key Discipline of Cell Biology, Fourth Military Medical University, Xi'an, China

${ }^{*}$ Corresponding authors: J-L Jiang or Z-N Chen, Department of Cell Biology, Cell Engineering Research Centre, State Key Laboratory of Cancer Biology, State Key Discipline of Cell Biology, Fourth Military Medical University, Xi'an 710032, China. Tel: + 8629 847745477; Fax: + 8629 83293906; E-mail: jiangj! @fmmu.edu.cn (JLJ) or znchen@fmmu.edu.cn (ZNC)

${ }^{2}$ These authors contributed equally to this work.

Keywords: CD147; unfolded protein response (UPR); hepatocellular carcinoma (HCC); Bip; apoptosis; chemosensitivity

Abbreviations: UPR, unfolded protein response; HCC, hepatocellular carcinoma; ERSE, endoplasmic reticulum stress response element; ER, endoplasmic reticulum; ERS, endoplasmic reticulum stress; IRE1, inositol-requiring enzyme 1; PERK, kinase-like endoplasmic reticulum kinase; ATF6, activating transcription factor 6; Tm, tunicamycin; Tg, thapsigargin; siRNA, small interfering RNA; shRNA, small hairpin RNA; Topo II, topoisomerase II

Received 21.12.11; revised 15.3.12; accepted 30.3.12; Edited by M Piacentini; published online 18.5 .12
} 
NO/cGMP-mediated negative regulation of store-operated $\mathrm{Ca}^{2+}$ entry, thus increasing the intracellular level of $\mathrm{Ca}^{2+}$ level in HCC cells. ${ }^{21,22}$ The disruption of intracellular calcium homeostasis can disturb ER function and induce ERS. ${ }^{23}$ These cellular responses, which are collectively referred to as the UPR, operate to augment the ER capacity to refold and degrade aberrant proteins. ${ }^{1}$ The role of CD147 in HCC progression and its role in intracellular $\mathrm{Ca}^{2}{ }^{+}$level changes in HCC cells suggest that CD147 may be involved in the UPR in human $\mathrm{HCC}$, and a detailed investigation to determine the precise mechanism of this phenomenon is required. In this study, we demonstrated that a cancer biomarker, CD147, functions as a novel UPR transducer to increase transcription of Bip in response to ERS, thus promoting HCC cell survival and decreasing chemosensitivity.

\section{Results}

Bip expression is positively associated with CD147 in human HCC tissues. As Bip is the major UPR marker, we examined the expression relevance of Bip and CD147 in human HCC tissues. Tumor specimens from patients who underwent hepatectomies before adjuvant therapy, including radiation and chemotherapy, and normal specimens from healthy liver transplant donors were examined using immunohistochemistry to determine CD147 and BiP expression in human HCC tissues. Compared with normal liver tissue in which CD147 was weakly expressed in 3 of the 42 normal liver tissues (7.1\%; Table 1a), CD147 expression was significantly increased in HCC tissues $(Z=-7.002$, $P=0.000)$ for 73 of $98(74.5 \%$; Table 1 a) cases of HCC tissues, including well, moderately and poorly differentiated tissues expressing CD147. These results further confirm the conclusion that CD147 is a cancer biomarker. ${ }^{24}$ While the expression of the ER-resident chaperone Bip was undetectable by immunohistochemistry in all 42 normal liver tissue samples (Table 1a), Bip was expressed in the majority of the 98 hepatoma tissues (83.7\%; Table 1a). These results reveal that Bip is highly elevated in human HCC tissues compared with normal liver tissues $(Z=-7.179, P=0.000)$, which suggested that the UPR is activated in human HCC tissues. Furthermore, the Spearman's rank correlations test demonstrated that Bip expression was positively associated with CD147 in human HCC tissues $\left(r_{\mathrm{s}}=0.535, P=0.000\right.$; Table 1b; Supplementary Figure S1), revealing that CD147 may function during the induction of the UPR.

CD147 promotes Bip expression in response to ERS in human HCC cells. To follow the kinetics of CD147 and Bip expression in response to ERS, 7721 and HepG2 human HCC cells were exposed to the ER stressors (i.e., reagents that induce ERS), tunicamycin ( $T m$, an inhibitor of $\mathrm{N}$-glycosylation) and thapsigargin (Tg, an inhibitor of ER $\mathrm{Ca}^{2+}$-ATPase). A relatively low level of CD147 and Bip expression was detected before $\mathrm{Tm}$ or $\mathrm{Tg}$ treatment. However, upon ER stressor treatment, there was a substantial increase in CD147 and Bip protein levels in a timedependent manner (Figure 1a).

As the ER is a key organelle for protein synthesis, ERS may induce the accumulation of misfolded proteins in the ER. ${ }^{25}$ To examine whether the increased CD147 after ER stressors treatment is misfolded protein accumulated in the ER or is properly folded protein mostly expressed at the cell surface, we tested the localization of CD147 in 7721 cells after ER stressors treatment. Immunofluorescence assay demonstrated that the increased CD147 was mostly localized on the HCC cell membrane (Figure 1b). This indicated that the increase of CD147 upon ER stressor treatment is not due to the accumulation of misfolded CD147 in the ER.

To directly detect whether Bip expression was correlated with CD147 expression, we used small interfering RNA (siRNA) to knockdown CD147 in 7721 cells. CD147 siRNA led to a marked reduction of CD147 levels in 7721 cells (Figure 1c). When the cells were stimulated with $\mathrm{Tm}$ or $\mathrm{Tg}$ for 18h, CD147 siRNA markedly diminished Bip mRNA and protein expression levels in 7721 cells (Figures 1c and d). As CD147 is a principal marker of different types of cancers, we further detected the induction of Bip by CD147 in MDA-MB231 human breast cancer cells (MM231) and A549 human lung adenocarcinoma cells after $\mathrm{Tm}$ or $\mathrm{Tg}$ treatment.

Table $1 \quad$ CD147 and Bip expression in human liver tissues

\begin{tabular}{|c|c|c|c|c|c|c|c|}
\hline & \multirow[b]{2}{*}{ Cases } & \multicolumn{4}{|c|}{ CD147 (\%) } & \multicolumn{2}{|c|}{ Bip (\%) } \\
\hline & & Negative & Weak & Moderate & Strong & Negative & Positive \\
\hline \multicolumn{8}{|l|}{ (a) } \\
\hline Normal tissue & 42 & $39(92.9)$ & $3(7.1)$ & $0(0.0)$ & $0(0.0)$ & $42(100.0)$ & $0(0.0)$ \\
\hline Hepatoma & 98 & 25 (25.5) & $40(40.8)$ & $26(26.5)$ & $7(7.1)$ & 16 (16.3) & $82(83.7)$ \\
\hline High differentiate & 4 & $1(25.0)$ & $2(50.0)$ & $1(25.0)$ & $0(0.0)$ & $1(25.0)$ & $3(75.0)$ \\
\hline Mediate differentiate & 77 & $18(23.4)$ & $31(40.3)$ & $21(27.3)$ & $7(9.1)$ & $12(15.6)$ & $65(84.4)$ \\
\hline \multirow[t]{2}{*}{ Low differentiate } & 17 & $6(35.3)$ & $7(41.2)$ & $4(23.5)$ & $0(0.0)$ & $3(17.6)$ & $14(82.4)$ \\
\hline & & \multicolumn{4}{|c|}{ CD147 (\%) } & \multirow[b]{2}{*}{$\mathbf{r}_{\mathbf{s}}$} & \multirow[b]{2}{*}{$P$-value } \\
\hline Bip & Cases & Negative & Weak & Moderate & Strong & & \\
\hline \multicolumn{8}{|l|}{ (b) } \\
\hline- & $16(16.3)$ & $14(87.5)$ & 2 (12.5) & $0(0.0)$ & $0(0.0)$ & 0.535 & 0.000 \\
\hline+ & $82(83.7)$ & $11(13.4)$ & $38(46.3)$ & $26(31.7)$ & $7(8.5)$ & & \\
\hline
\end{tabular}

${ }^{a}$ Comparison of CD147 and Bip expression in normal tissues and HCC, including well, moderately and poorly differentiated tissues. $P$-values were calculated by the Mann-Whitney $U$-test. (CD147: $Z=-7.002, P=0.000$; Bip: $Z=-7.179, P=0.000$ )

${ }^{\mathrm{b}}$ The correlation of Bip and CD147 expression levels in human HCC tissues. $P$-values were calculated by Spearman's rank correlations test $(n=98)$ 
a

7721

\begin{tabular}{lllll}
\multicolumn{6}{c}{$\operatorname{Tm}$} \\
\hline 0 & 6 & 12 & 18 & $24(\mathrm{~h})$
\end{tabular}

CD147

Bip

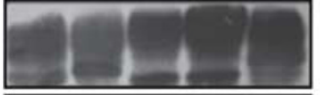

$\beta$-actin

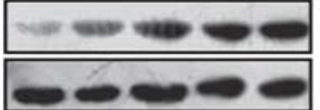

HepG2
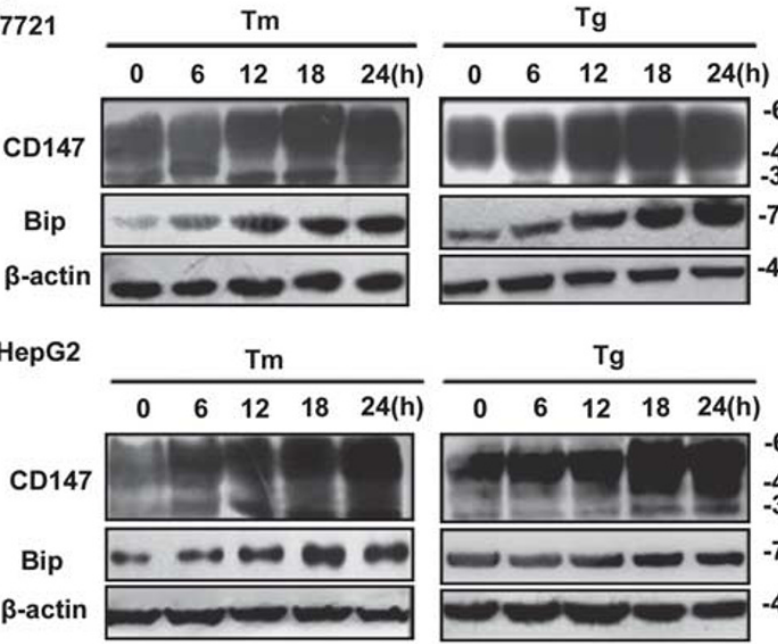

$-66 \mathrm{kDa}$

$-43 \mathrm{kDa}$

$35 \mathrm{kDa}$

$-78 \mathrm{kDa}$

$42 \mathrm{kDa}$
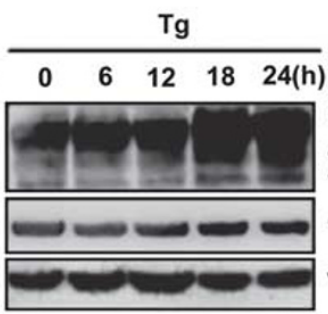

$-66 \mathrm{kDa}$
$-43 \mathrm{kDa}$
$-35 \mathrm{kDa}$
$-78 \mathrm{kDa}$
$-42 \mathrm{kDa}$

c

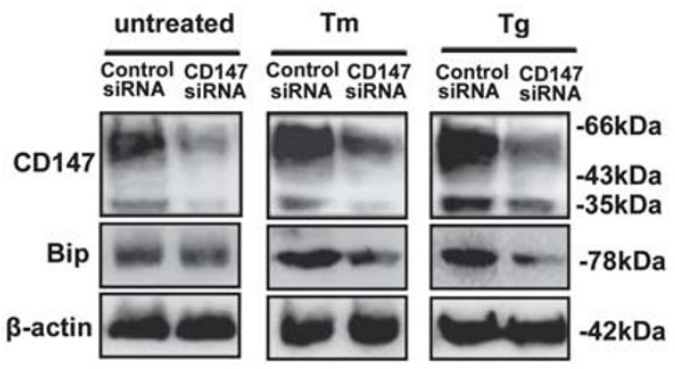

e

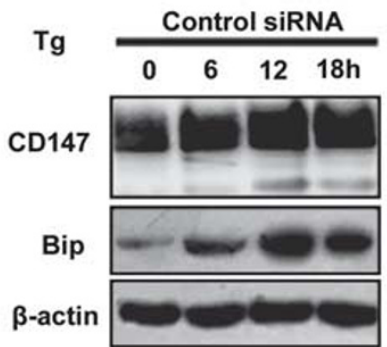

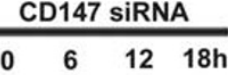

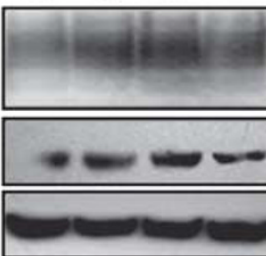

b

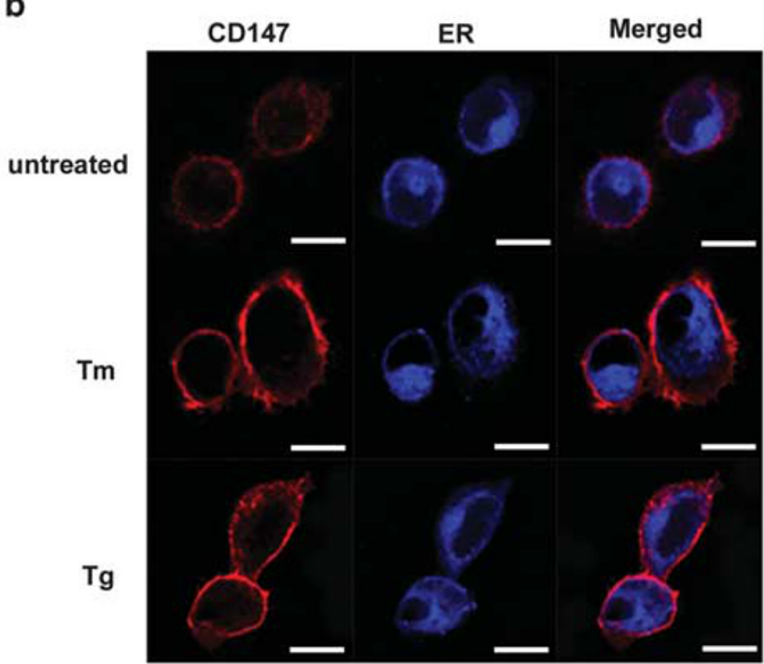

d
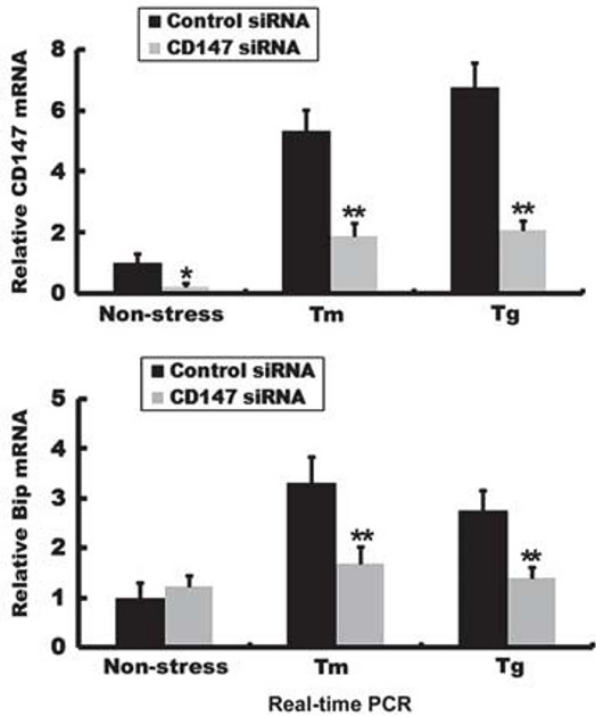

Figure 1 CD147 promotes Bip expression in response to ERS in human HCC cells. (a) 7721 and HepG2 cells were treated with $1 \mu \mathrm{M} \mathrm{Tm}$ or $100 \mathrm{nM} \mathrm{Tg} \mathrm{for} 0,6,12,18$, $24 \mathrm{~h}$. Western blot was used to examine CD147 and Bip protein levels. (b) Immunofluorescence was used to assess the localization of CD147 in 7721 cells after treatment with $\operatorname{Tm}$ or $\mathrm{Tg}(\mathrm{Bar}=10 \mu \mathrm{m})$. (c) The 7721 cells were treated with CD147 siRNA, incubated for $24 \mathrm{~h}$ and then stimulated with $\mathrm{Tm}$ or Tg for $18 \mathrm{~h}$. Western blot was performed to examine the CD147 and Bip protein levels. Control siRNA transfected 7721 cells were used as a negative control. (d) Real-time PCR was performed to examine the CD147 and Bip mRNA levels. (e) The 7721 cells were treated with CD147 siRNA, incubated for $24 \mathrm{~h}$ and then stimulated with $\mathrm{Tg}$ for $0,6,12$ and $18 \mathrm{~h}$. Western blot was performed to examine the decrease of CD147 and Bip by CD147 siRNA for various time periods. Bars represent each sample performed in triplicate, and the error bars represent the standard deviations. ${ }^{*} P<0.05,{ }^{\star *} P<0.01$, by Student's $t$-test

As shown in Supplementary Figure S2, Bip expression significantly diminished in CD147 siRNA transfected cells compared with corresponding control siRNA transfected cells. Together, these results suggest that CD147 may play roles in Bip induction in response to ERS.

Next, to more directly assess the requirement of CD147 in Bip induction, we monitored the decrease of CD147 and Bip by CD147 siRNA for various time periods. After the exposure to $\mathrm{Tg}$ for $6 \mathrm{~h}$, Bip protein levels in CD147 siRNA transfected 7721 cells were induced to similar levels, as in control siRNA transfected 7721 cells. However, at $12 \mathrm{~h}$ and later, the level of Bip protein decreased by almost $50 \%$ in CD147 siRNA transfected 7721 cells compared with the control siRNA transfected 7721 cells at each time point (Figure 1e). This suggested that CD147 is important for the induction of Bip expression during ERS, particularly during the late phase of UPR signaling. This may be related to the transcriptional induction of CD147 mRNA after ERS.

CD147 induces nuclear localization of TFII-I and the binding of TFII-I to the Bip promoter to increase the transcription of Bip in response to ERS in human HCC cells. IRE1, PERK and ATF6 are three key factors that trigger UPR signaling and subsequently promote the expression of Bip. ${ }^{12-14}$ Therefore, we examined the expression and phosphorylation levels of these genes in CD147 
siRNA transfected 7721 cells. Unexpectedly, CD147 siRNA did not significantly change these proteins expression levels with or without $\mathrm{Tg}$ treatment (Figure 2a).

We then tested the expression levels of two major transcription factors, TFII-I and YY1, which can directly bind to the ERS elements (ERSEs) in the Bip promoter in HeLa cells. ${ }^{26}$ We observed that CD147 siRNA did not decrease the YY1 expression levels in 7721 cells (Figure 2b). However, CD147 siRNA markedly diminished the $\mathrm{p}$-TFII-I levels after Tg treatment (Figure 2b). These results indicate that CD147 may induce tyrosine phosphorylation of TFII-I in response to ERS.
Transcription factors might play roles via their translocation from the endochylema to the nucleus. Thus, cytoplasmic and nuclear proteins were extracted and analyzed. The results showed that TFII-I phosphorylation significantly decreased in the nucleus of CD147 siRNA transfected 7721 cells (Figure 2c). Immunofluorescent staining for phosphorylated TFII-I further confirmed that in CD147 siRNA transfected 7721 cells, the phosphorylated TFII-I fluorescence was weak and predominantly localized to the cytoplasm (Figure 2d). To further confirm the above results, we transfected CD147 expression plasmid into 7721 cells to obtain CD147
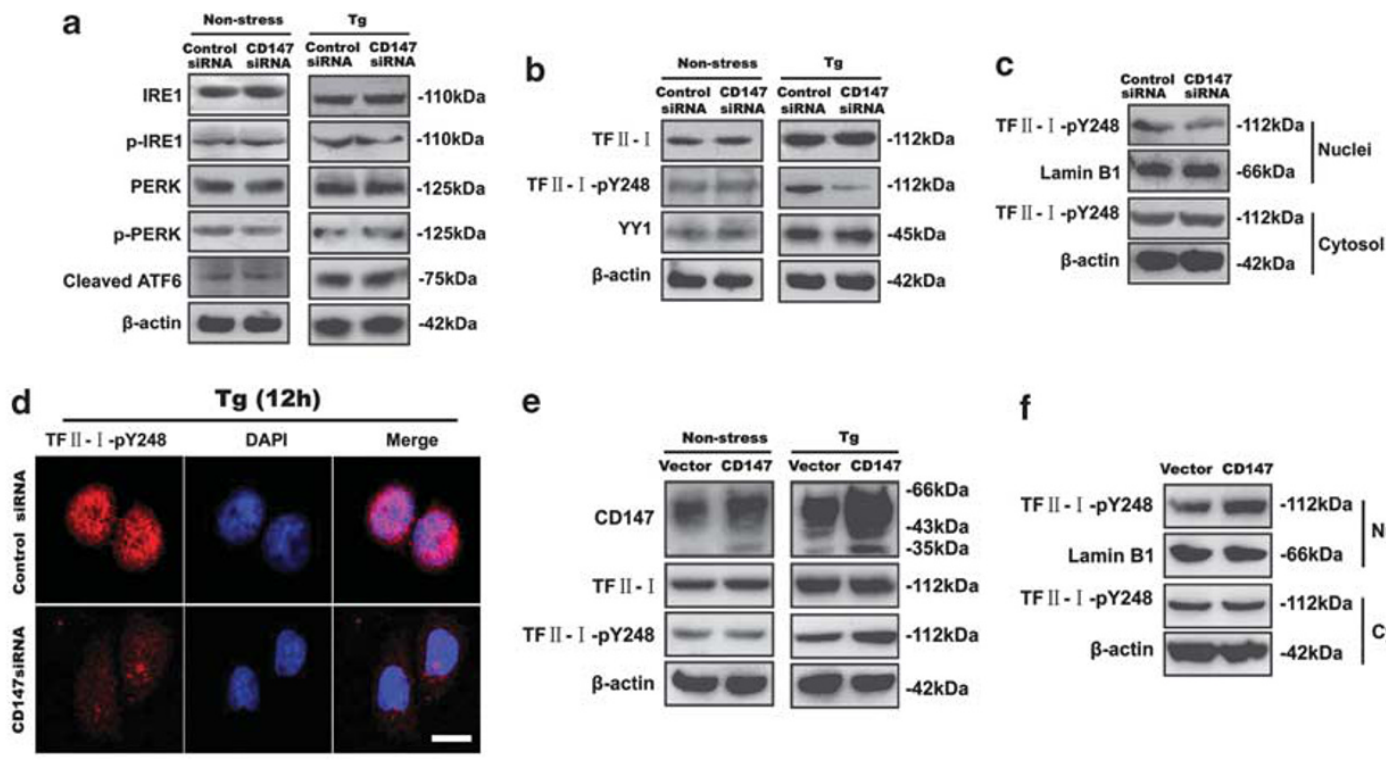

e
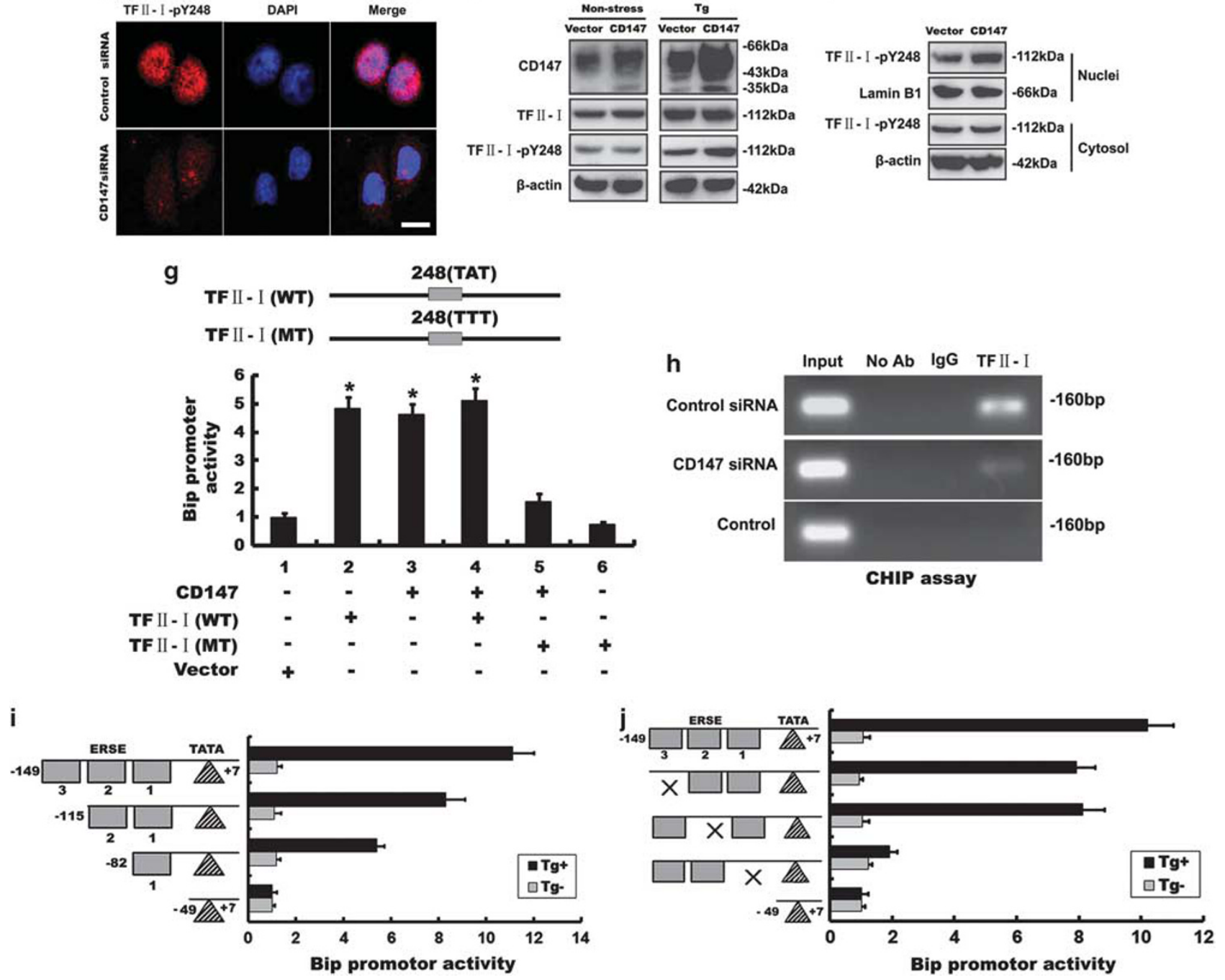

Cell Death and Differentiation 
overexpressing cells (Figure 2e). As shown in Figure 2e, after Tg treatment, the p-TFII-I levels were significantly increased in CD147 overexpressing 7721 cells. Nuclear and cytoplasmic protein extraction results showed that TFII-I phosphorylation markedly increased in the nucleus of CD147 overexpressing 7721 cells after $\mathrm{Tg}$ treatment (Figure 2f). Collectively, these results indicate that CD147 induces TFII-I tyrosine phosphorylation and nuclear localization in response to ERS in human HCC cells.

To detect whether CD147-promoted Bip induction was mediated through TFII-I, a Bip $(-149 /+7) /$ Luc promoter construct was co-transfected with CD147 or TFII-I expression plasmids, alone or in combination. We observed that either CD147 or TFII-I alone stimulated Bip $(-149 /+7) /$ LuC reporter gene activity after $\mathrm{Tg}$ treatment. However, the combination of both resulted in no further enhancement (Figure $2 \mathrm{~g}$, lanes 2-4). This result may indicate that CD147 and TFII-I perform functional overlapping roles on enhancing the Bip promoter activity in ERS. The functional overlap suggests a molecular link between CD147 and TFII-I in Bip induction. The tyrosine phosphorylation motif at residues 248 of TFII-I is critical for transcriptional activity of TFII-I. ${ }^{27}$ To further address whether TFII-I is required for CD147promoted Bip induction in ERS, we co-transfected the Bip $(-149 /+7) /$ Luc promoter construct and the CD147 expression plasmid together with either TFII-I (WT) or Tyr248 mutated TFII-I (MT) expression plasmids. As shown in Figure $2 \mathrm{~g}$ in lanes 4 and 5, compared with CD147 cotransfected with TFII-I (WT), CD147 co-transfected with TFII-I (MT) suppressed Bip promoter activation. The inhibitive effect of TFII-I (MT) on Bip promoter activation indicate that CD147promoted Bip induction is induced by TFII-I.

To directly elucidate the effects of CD147 on the binding of TFII-I to the Bip promoter, chromatin immunoprecipitation (ChIP) assays were performed in Tg-treated CD147 siRNA transfected 7721 cells. We found that CD147 siRNA markedly decreased binding activity of TFII-I to the Bip promoter (Figure 2h). These findings suggested that CD147 may increase binding activity of TFII-I to the Bip promoter.

Bip promoter contains multiple ERSE copies, with the consensus sequence CCAAT(N9)CCACG located upstream of the TATA element. ${ }^{26}$ To map the TFII-I target sites within the Bip promoter, the full-length TFII-I expression plasmid was co-transfected into HEK-293 cells with a series of $5^{\prime}$-deletion mutants of the human Bip promoter, which was linked to the Luc promoter gene. Compared with the reporter activity of a minimal promoter containing essentially only the TATA element of the Bip promoter, we observed an almost 10-fold reporter activity of the $-149 /+7$ Luc promoter, which contains three ERSE copies cloned upstream of the TATA element, an eightfold reporter activity of the $-115 /+7$ Luc promoter, which contains two ERSE copies and a fivefold reporter activity of the $-82 /+7$ Luc promoter, which contains one ERSE copy (Figure 2i). Next, we mutated the three ERSEs in the Bip promoter. Mutation of ERSE3 alone reduced the TFII-I-induced reporter activity by $\sim 22 \%$ compared with the wild-type promoter, mutation of ERSE2 alone reduced the TFII-I-induced reporter activity by $\sim 20 \%$, and mutation of ERSE1 alone decreased the TFII-I-induced reporter activity to $<70 \%$ (Figure 2j). This more severe effect suggests that the TFII-I targets all three ERSEs sites of Bip promoter but preferentially acts on the ERSE1 site.

\section{FAK and Src mediate CD147-promoted Bip transcription} in response to ERS in human HCC cells. TFII-I is phosphorylated at Tyr248 in a FAK-Src-dependent fashion. ${ }^{18}$ To identify whether FAK and Src is involved in CD147induced TFII-I tyrosine phosphorylation, the expression and phosphorylation levels of FAK and Src were tested. CD147 siRNA markedly decreased p-FAK and p-Src levels in 7721 cells, in both the absence and presence of Tg (Figures $3 \mathrm{a}$ and b). These data indicate that CD147 may activate the FAK-Src signaling pathway with or without Tg treatment.

We next used the FAK inhibitor PF573288 and the Src inhibitor S2075 to examine whether CD147-induced Bip induction is dependent on the FAK-Src signaling pathway after Tg treatment. The results showed that CD147 siRNA did not further reduce $\mathrm{p}$-TFII-I and Bip levels following the FAK or the Src inhibitor treatment (Figure $3 \mathrm{c}$ ). Our previous results demonstrate that CD147 facilitates FAK phosphorylation at Tyr397 by interacting with $\alpha 3 \beta 1$ integrin. ${ }^{22}$ We confirmed these results by the observation that CD147 siRNA did not further reduce Bip levels after blocking $\alpha 3 \beta 1$ integrin with the addition of $10 \mu \mathrm{M}$ integrin $\beta 1$ antibody (Figure $3 \mathrm{~d}$ ). These

Figure 2 CD147 induces nuclear localization of TFII-I and the binding of TFII-I to the Bip promoter to increase the transcription of Bip in response to ERS in human HCC cells. (a) The 7721 cells were treated with CD147 siRNA, incubated for $24 \mathrm{~h}$ and then were either left untreated or treated with $\mathrm{Tg}$ for $18 \mathrm{~h}$. Western blot was used to examine the expression levels of IRE1, p-IRE1, PERK, p-PERK and cleaved ATF6. (b) The expression levels of YY1, TFIIII and p-TFII-I were examined in CD147 siRNA transfected 7721 cells either untreated or treated with Tg for $18 \mathrm{~h}$. (c) The TFII-I phosphorylation levels were tested in the nuclear and cytoplasmic fractions of CD147 siRNA transfected 7721 cells treated with $\mathrm{Tg}$ for $18 \mathrm{~h}$. Protein expression levels in the nuclear and cytosolic fractions were normalized to Lamin B1 and $\beta$-actin, respectively. (d) Immunofluorescence staining was performed to observe the location of phosphorylated TFII-I in CD147 siRNA transfected 7721 cells treated with Tg for $18 \mathrm{~h}$. $(B a r=10 \mu \mathrm{m})$. (e) The 7721 cells were transfected with CD147 expression plasmid (CD147-pcDNA3.1), incubated for $48 \mathrm{~h}$ and then were either left untreated or treated with Tg for $18 \mathrm{~h}$. Western blot was used to examine the expression levels of CD147, TFII-I and p-TFII-I. (f) The TFII-I phosphorylation levels were tested in the nuclear and cytoplasmic fractions of CD147 overexpressing 7721 cells treated with $\mathrm{Tg}$ for $18 \mathrm{~h}$. $(\mathbf{g})$ The activity of the Bip promoter was tested by Luciferase reporter assay in HEK-293 cells. Bip promoter linked to the Luc promoter gene was co-transfected with either CD147-pcDNA3.1, or TFII-I (WT)-pcDNA3.1, or Tyr248 mutated TFII-I (MT)-pcDNA3.1 expression plasmids, or pcDNA3.1 empty vector, alone and in combination. Five hours after transfection, cells were treated with $\mathrm{Tg}$ for $18 \mathrm{~h}$, and the luciferase activity was measured. (h) ChIP assay using antibody against TFII-I was performed in Tg-treated CD147 siRNA transfected 7721 cells. The no antibody (no Ab) was used as a blank control, the normal mouse IgG was used as a negative control and input indicates $5 \%$ input DNA, a positive amplification control. The 160-bp fragment was amplified by the Bip promoter-specific primers contains the TFII-I binding sequence, whereas the control PCR reactions generated a 160-bp fragment does not contain any TFII-I binding sequence. (i) The TFII-I expression plasmid was co-transfected with a series of $5^{\prime}$-deletion mutants of the human Bip promoter or (j) with a series of three ERSE mutations of the human Bip promoter linked to the Luc promoter gene into HEK-293 cells. Five hours after transfection, cells were treated with $\mathrm{Tg}$ for $18 \mathrm{~h}$, and the luciferase activity in each sample was measured. Bars represent each sample performed in triplicate, and the error bars represent the standard deviations. ${ }^{*} P<0.05$, by the ANOVA analysis or Student's t-test 
a
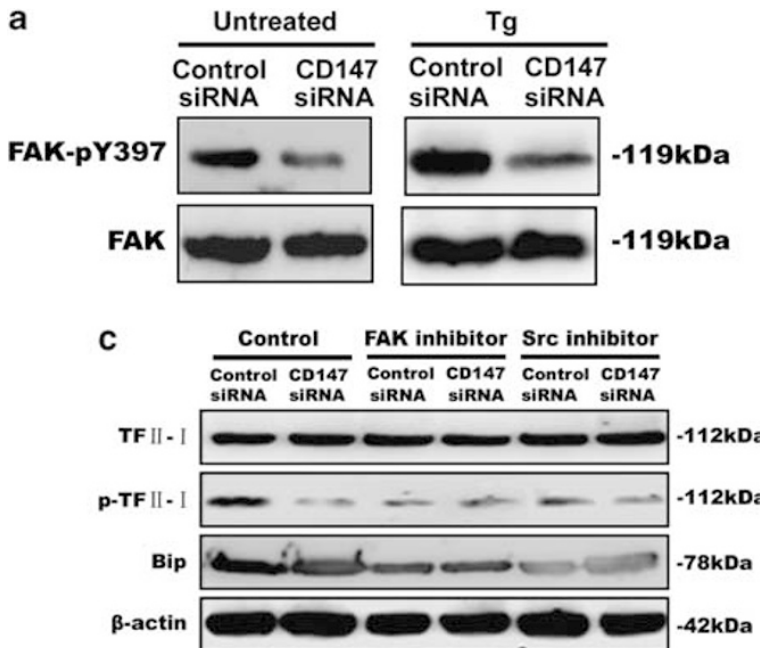

b

Control CD147

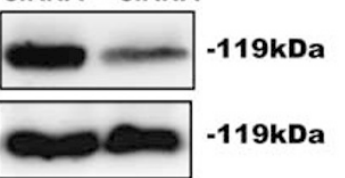
SiRNA SIRNA
$\mathrm{Tg}$

Control CD147

SIRNA SiRNA

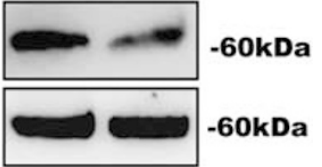

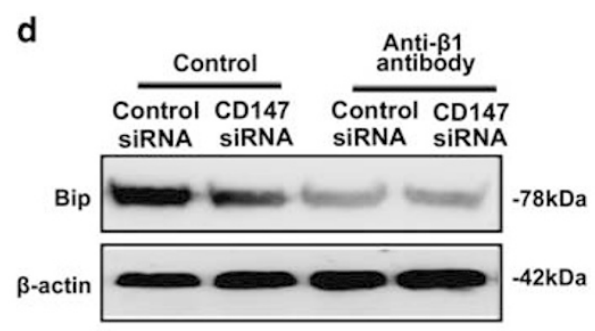

Figure 3 FAK and Src mediate CD147-induced Bip induction in response to ERS in human HCC cells. (a) The 7721 cells were treated with CD147 siRNA, incubated for $24 \mathrm{~h}$ and then were either left untreated or treated with $\mathrm{Tg}$ for $18 \mathrm{~h}$. Western blot was used to test the FAK expression and phosphorylation levels. (b) The Src expression and phosphorylation levels were tested in CD147 siRNA transfected 7721 cells either untreated or treated with Tg for $18 \mathrm{~h}$. (c) After FAK inhibitor PF573288 or Src inhibitor S2075 treatment, the protein levels of TFII-I, p-TFII-I and Bip were examined. (d) Bip protein levels were tested after integrin $\beta 1$ antibody treatment

results suggest that $\alpha 3 \beta 1$ integrin, FAK and Src are involved in CD147-induced Bip expression.

CD147 inhibits ERS-induced HCC cell apoptosis and decreases chemosensitivity by inducing Bip expression. Due to its anti-apoptotic property, Bip functions as a cytoprotective protein in stressed cells by activating the UPR. ${ }^{25}$ Because CD147 increases Bip during ERS, CD147 may protect cells from ERS-induced cell apoptosis. To test this hypothesis, cell death was examined by microscope (Supplementary Figure S3) and TUNEL staining (Figure 4a), and then quantified by Annexin V/PI (propidium iodide) double staining (Figure $4 \mathrm{~b}$ ). The results demonstrated that CD147 siRNA caused less resistance to ERS-induced cell apoptosis. To further confirm that Bip is involved in this process, Bip siRNA was used (Supplementary Figure S4). Annexin V/PI double staining demonstrated that CD147 siRNA did not further increase the apoptotic cells following Bip depletion (Figure 4c). To further confirm these results, the activation of caspase-3 was detected. The results showed that CD147 siRNA increased caspase-3 activation during ERS and did not further increase caspase-3 activation following Bip depletion (Figure 4d). From the above results, we conclude that CD147 may protect HCC cells from ERSinduced apoptosis, and Bip is required in this process.

ADR (Adriamycin) and VP-16, two widely used anti-cancer drugs, were used to directly test whether CD147 protects HCC cells resistant to drug by activating UPR. Colony formation assays showed that ADR and VP-16 induced cell death in a dose-dependent manner in Tg-treated 7721 cells. Further, ADR and VP- 16 caused significantly more cell death in CD147 siRNA transfected 7721 cells than control siRNA transfected 7721 cells at every concentration measured (Figure 4e). CD147 siRNA, however, did not further increase cell death following Bip depletion after ADR or VP-16 treatment (Figure 4f). These results show that CD147 causes a statistically significant decrease in drug sensitivity by inducing Bip during ERS.

By inhibiting cell apoptosis and chemosensitivity, CD147 decreases the survival rate in nude mice bearing established orthotopic HCC. An orthotopic nude mouse HCC model was used to analyze the in-vivo function of CD147 in the ERS state. The CD147 small hairpin RNA (shRNA) stably transfected 7721 cells ( 7721 -shCD147 cells) and a control shRNA stably transfected 7721 cells $(7721$-snc cells; Supplementary Figure S5) were orthotopically inoculated in nude mice to establish orthotopic HCC. ${ }^{28}$ Immunohistochemistry of tumor tissues revealed CD147 shRNA downregulated Bip expression of established orthotopic HCC tissues (Figure 5a). Real-time PCR further confirmed these results (Figure 5b). TUNEL staining showed that CD147 shRNA increased apoptotic cells of established orthotopic HCC tissues (Figure 5a).

We next tested the chemosensitivity of established orthotopic HCC in a nude mouse model. Two GFP stably expressed cell lines, 7721-shCD147 and 7721-snc cells stably expressing GFP, were established by lentiviral infection. We observed a strong correlation between GFP activity and cell number (Figure $5 \mathrm{c}$ ). Then the cells were orthotopically inoculated in nude mice to establish GFPexpressing HCC. One day after implantation, every cell line inoculated nude mouse was randomized into one of three groups: the ADR treatment group, the $0.9 \% \mathrm{NaCl}$ treatment group (negative control) and the untreated group (blank control). Serial imaging showed a gradual GFP signal increase in all of the groups, and significant GFP signal differences were found between the groups $(P=0.000$, $F=961.759$, ANOVA; Figure 5d). There was no significant GFP signal difference between the blank control and the corresponding negative control groups (LSD-t-test), whereas the GFP signal was significantly lower in the ADR-treated 


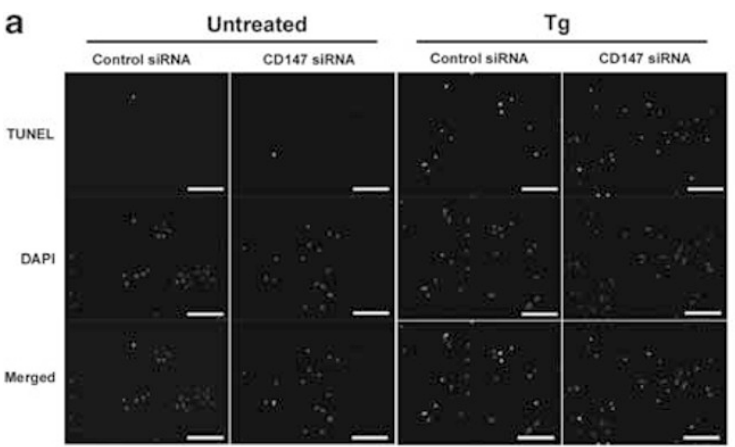

C Tg

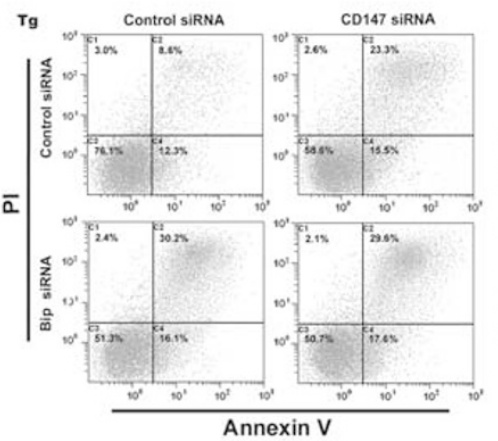

b

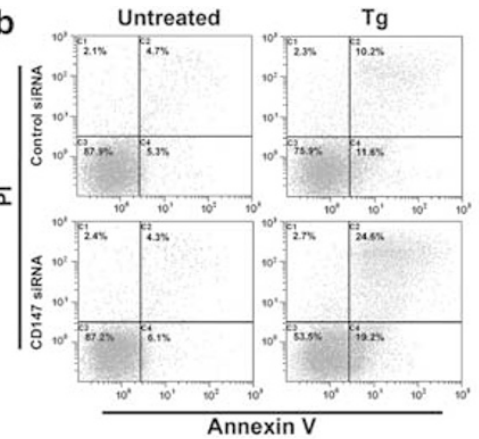

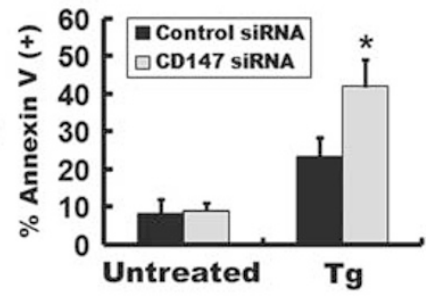

d
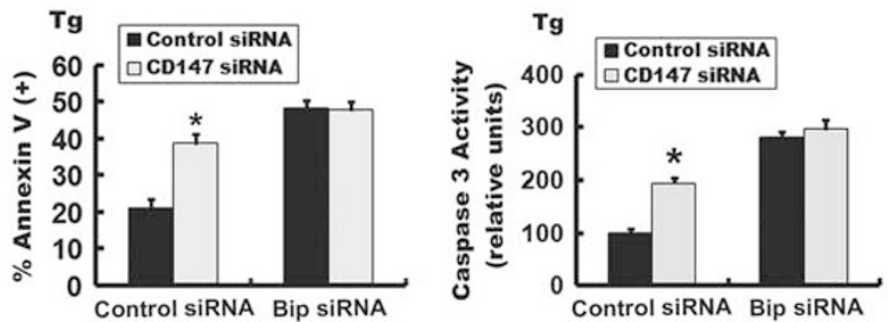

e
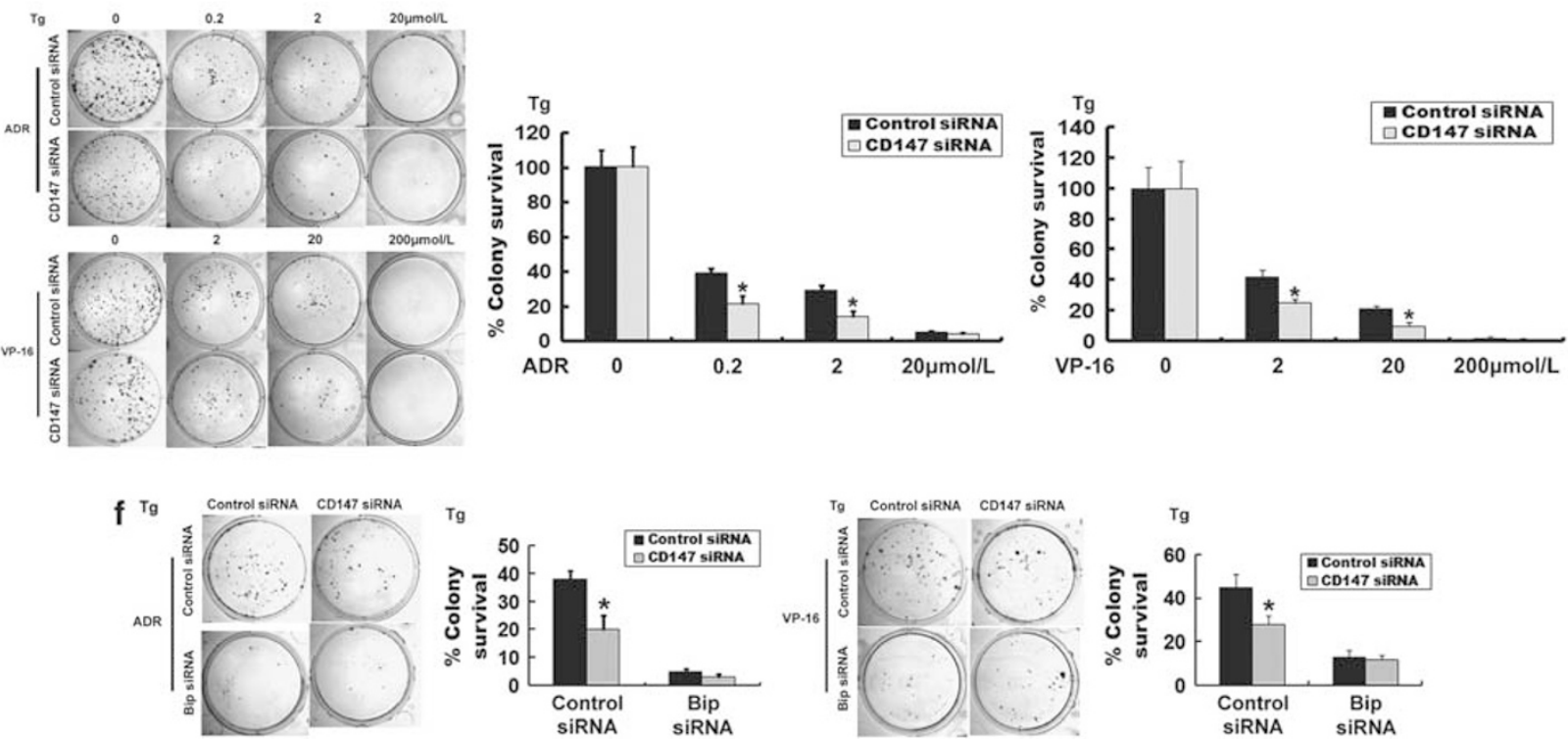

Figure 4 CD147 inhibits ERS-induced HCC cell apoptosis and decreases chemosensitivity by inducing Bip expression. (a) The 7721 cells were treated with CD147 siRNA, incubated for $24 \mathrm{~h}$, and then were either left untreated or treated with Tg for $18 \mathrm{~h}$. Cell death was tested by TUNEL staining $(B a r=100 \mu \mathrm{m})$ and $(\mathbf{b})$ quantified by Annexin V/PI double staining. (c) The 7721 cells were treated with CD147 or Bip siRNA, alone and in combination, and then were stimulated with Tg for $18 \mathrm{~h}$. Annexin V/PI double staining was performed to detect the apoptotic cells. (d) Caspase-3 assay kit was used to detect the caspase-3 activity. (e) A range of concentrations of ADR and VP-16 were used to evaluate the drug sensitivity of the CD147 siRNA transfected 7721 cells by colony formation assays. (f) The 7721 cells were treated with CD147 or Bip siRNA, alone and in combination, and then were stimulated with Tg for $18 \mathrm{~h}$. ADR $(0.2 \mu \mathrm{g} / \mathrm{ml})$ and VP-16 $(2 \mu \mathrm{g} / \mathrm{ml})$ were used to evaluate drug sensitivity by colony formation assays. Bars represent each sample performed in triplicate, and the error bars represent the standard deviations. ${ }^{*} P<0.05$, by Student's t-test

7721 -shCD147 cells-implanted group (32.7\% inhibitory rate) than in the ADR-treated 7721 -snc cells-implanted group (16.2\% inhibitory rate). These results demonstrate that CD147 shRNA increases sensitive of HCC to ADR in vivo.

We also investigated the survival rate in nude mice with established orthotopic hepatomas after treatment with ADR
(Figure 5e). The blank control groups and negative control groups uniformly succumbed to HCC within 30 days. The infusion of ADR in the 7721-snc cells-implanted group yielded modest survival advantages, whereas treatment with ADR in the 7721-shCD147 cells-implanted group demonstrated enhanced efficacy, with all mice surviving $>25$ days. Overall, 
a

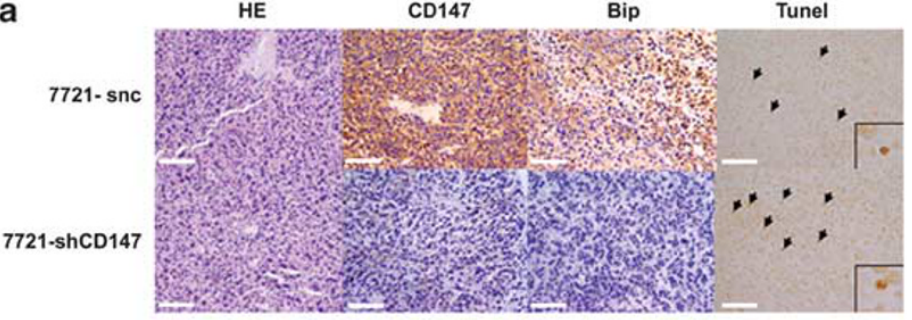

HE

CD147
GFP

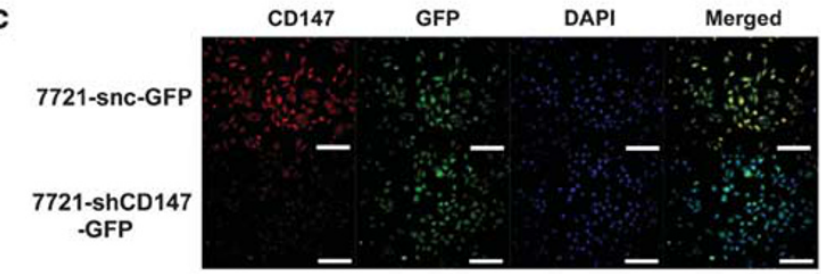

b

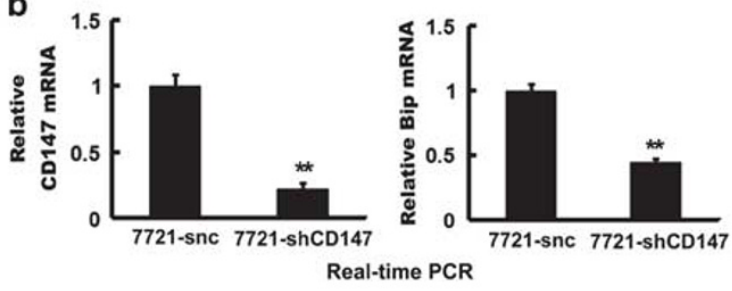

d
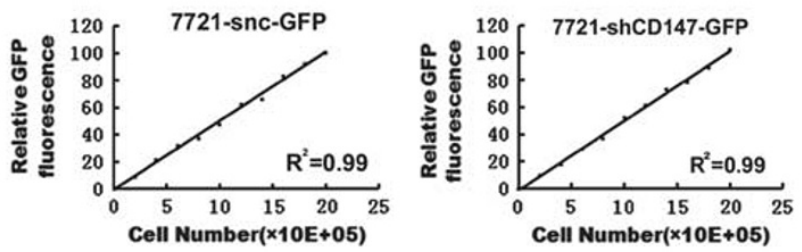

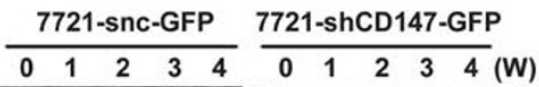
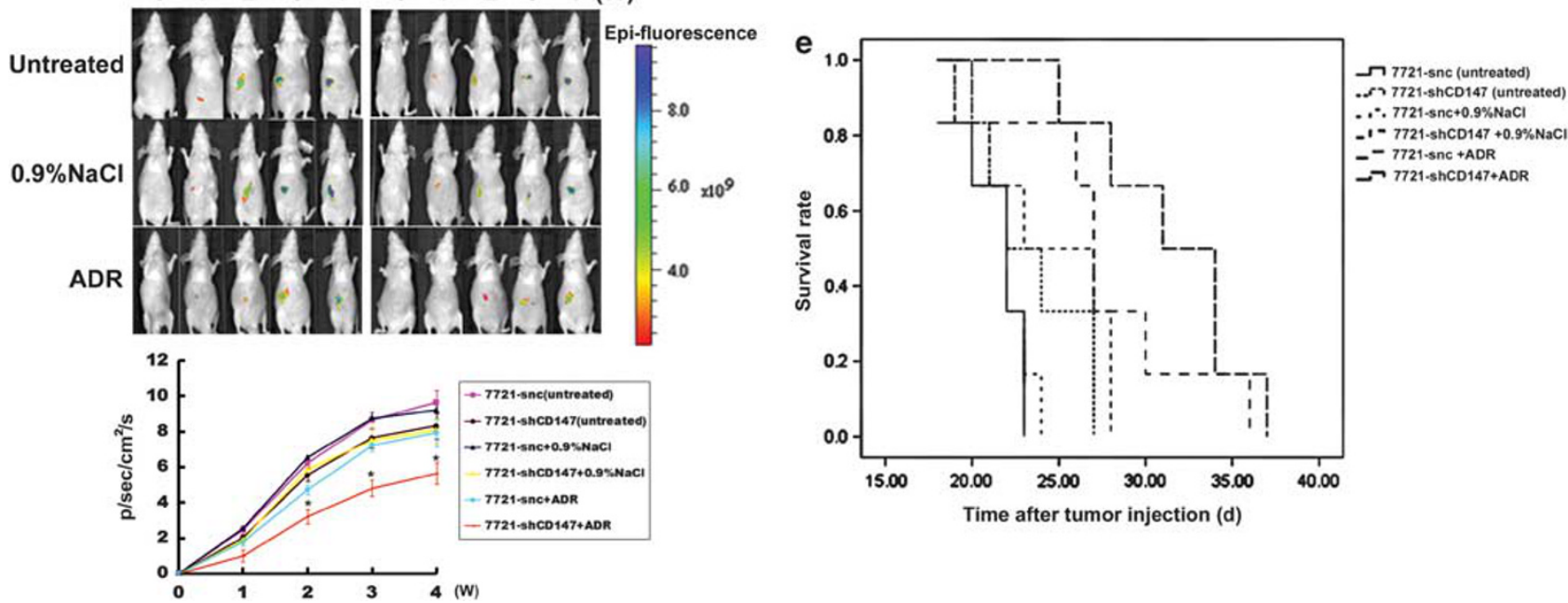

Figure 5 CD147 knockdown increases the survival rate in nude mice bearing established orthotopic HCC. (a) HCCs were established by orthotopically injecting $2 \times 10^{6}$ 7721-shCD147 cells into nude mice. After 45 days, the mice were killed, and their livers were dissected. Immunohistochemistry was performed to detect the protein expressions of CD147 and Bip proteins in tumor tissue. Tumor tissue apoptosis was tested by TUNEL staining (right). (b) Real-time PCR was used to test CD147 and Bip mRNA in established orthotopic HCC tissues. Bars represent the mean of each sample performed in triplicate, and the error bars represent the standard deviations. ${ }^{* \star} P<0.01$, by Student's $t$-test. (c) The reconstructed lentiviral vector p-WPT-GFP was stably transduced into 7721-shCD147 cells and 7721-snc cells to establish two GFP stably expressed cell lines. Immunofluorescence demonstrates the expression levels of CD147 (red) and GFP (green) in the two cell lines (Bar $=100 \mu \mathrm{m}$ ). A strong correlation exists between the cell number and GFP fluorescence intensity (7721-shCD147 cells, $R^{2}=0.99 ; 7721$-snc cells, $\left.R^{2}=0.99\right)$. (d) GFP-expressing HCCs were established by orthotopically injecting $2 \times 10^{6}$ GFP-expressing cells into nude mice. Tumor-bearing mice were treated with $2.5 \mathrm{mg} / \mathrm{kg} \mathrm{ADR}$ (three times, 7 days), or a similar volume of $0.9 \%$ $\mathrm{NaCl}$ solution (three times, 7 days, the negative control) or were untreated (blank control). The GFP signal was monitored at the indicated time points $(0,1,2,3,4$ weeks) after treatment. The color bars indicate photon counts relative to the pseudocolor for the adjacent panel images. Bars represent the mean of three samples, and the error bars represent the standard deviations. ${ }^{*} P<0.05$, by ANOVA analysis. (e) The survival rate of nude mice after treatment is illustrated by Kaplan-Meier curves. Six mice per treatment group pooled from three independent experiments are shown. Relevant $P$-values (log-rank test) are depicted for each group

these data indicate that CD147 inhibits chemosensitivity and decreases the survival rate in a nude mouse model of orthotopic HCC.

\section{Discussion}

The UPR, a pathway activated by the accumulation of misfolded secretory and membrane proteins in the ER, involves the transcriptional induction of genes that encode proteins, such as the ER chaperone, Bip, and selected transcription factors. ${ }^{29}$ UPR activation in cancer is an adaptive survival mechanism that protects cells from ERS-induced cell death. In this study, we characterized CD147 as a UPR inducer that increases Bip transcription, thus promoting human HCC cell survival and increasing chemoresistance in the ERS state.

As a key molecular chaperone within the ER, Bip is a marker of the UPR. ${ }^{25}$ In this study, we demonstrated that Bip expression is elevated in HCC biopsies and is positively associated with the cancer biomarker, CD147, in human HCC 
tissues. We next confirmed that CD147 promotes Bip expression in HCC cells in the context of ERS. By phosphorylating FAK and Src, CD147-enhanced tyrosine TFII-I phosphorylation at Tyr248 and induced the nuclear localization of $p$-TFII-I, subsequently enhanced the binding of TFII-I to the Bip promoter, resulting in the increased transcription of Bip in HCC (Figure 6).

TFII-I was first discovered as an initiator binding protein. ${ }^{30}$ Accumulating evidence suggests that TFII-I may have the capacity for multiple protein-protein interactions and, potentially, multiple protein-DNA interactions. Purified recombinant TFII-I isoforms bind directly to the Bip promoter ERSE. ${ }^{31}$ The stimulation of ERSE-mediated transcription by TFII-I requires the integrity of the consensus tyrosine phosphorylation site and the GGC sequence ERSE motif. The Bip promoter contains three ERSE copies, with each ERSE consisting of a tripartite structure: CCAAT(N9)CCACG, with $\mathrm{N}$ being an invariant region of $9 \mathrm{bp}$. The GGC sequence occurs once and sometimes twice within the $\mathrm{N} 9$ region of mammalian ERSEs. ${ }^{32}$ This study revealed that the three Bip promoter ERSEs are all needed in CD147-promoted, TFII-I-dependent Bip transcription in response to ERS, although ERSE1(-82/ $-50)$ is the most efficient target. TFII-I can associate with ATF6, and is required for the maximal stimulation of ERSE by ATF6. ${ }^{26}$ These two factors, together with $Y Y 1$, become a part of a multiprotein complex that binds to ERSE. ${ }^{26}$ Components of this multiprotein complex have the potential to serve as bridging proteins between the upstream ERSE regulatory elements and the basal transcriptional machinery.

The observation that either CD147 or TFII-I alone enhanced Bip promoter activity, and the combination of both resulted in no further enhancement may indicate an overlapping mechanism between CD147 and TFII-I in stimulating the Bip promoter in ERS. We speculated that in the absence of TFII-I, CD147 may promote Bip induction by activating other endogenous transcription factors. However, in the presence of TFII-I, CD147 appears to preferentially activate TFII-I to

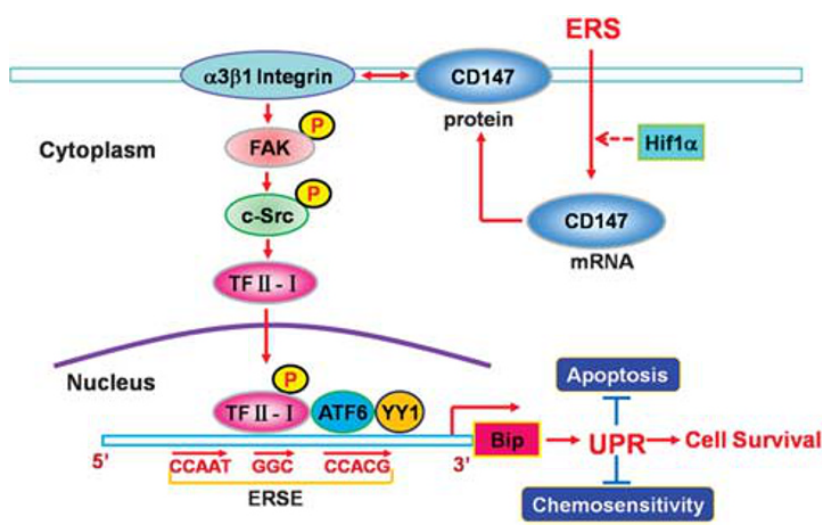

Figure 6 Putative mechanisms responsible for UPR activation by CD147 in human HCC cells. CD147 is induced during ERS in human HCC cells. By interacting with integrin $\alpha 3 \beta 1$, CD147 activates the FAK-Src signaling pathway, which promotes p-TFII-I nuclear translocation and the subsequent transcription of Bip via binding to the ERSE sites. By inhibiting ERS-induced cell apoptosis and decreasing chemosensitivity, this CD147-mediated signaling pathway may contribute to cell survival after ERS in human HCC cells promote Bip promoter activity and override other endogenous transcription factors. To test this hypothesis, we used Tyr248 mutated TFII-I (MT) expression plasmids, which lose the transcriptional activity on the Bip promoter. We observed that compared with CD147 co-transfected with TFII-I (WT), CD147 co-transfected with TFII-I (MT) suppressed Bip promoter activation. The inhibitive effect of TFII-I (MT) on CD147enhanced activation of Bip promoter confirmed that although other transcription factors might exist, CD147-promoted Bip induction is mainly induced by TFII-I in the presence of TFII-I. TFII-I as a multifunctional transcription factor widely exists in mammalian cells and its expression level markedly increases in response to ERS. ${ }^{26}$ Therefore, it is conceivable that CD147 activates TFII-I to promote Bip transcription is the most predominant pathway in CD147-promoted Bip induction in ERS. The competitive inhibition of TFII-I on other transcription factors involved in CD147-induced Bip transcription needs to be further investigated.

Our previous study demonstrated that by the interaction with integrin $\alpha 3 \beta 1$, CD147 may promote FAK phosphorylation at Tyr397. ${ }^{22}$ FAK autophosphorylation at Tyr397 increases FAK kinase activity and creates a high-affinity binding site for the $\mathrm{SH} 2$ domain of Src. Occupancy of the Src SH2 domain is thought to result in the activation of Src, suggesting a role for FAK upstream of $\mathrm{Src}^{24}$ We discovered that by mediating TFII-I tyrosine phosphorylation at Tyr248, Src is involved in CD147-induced TFII-I activation, and the consensus tyrosine phosphorylation site at residue 248 is required for TFII-I stimulation of the ERSE, which is consistent with previous findings. ${ }^{27}$

Tumor progression may occur as the result of anti-apoptotic mechanisms combined with a general tolerance to suboptimal microenvironmental conditions as a result of the cytoprotective components of the UPR. We demonstrated that by the activation of the UPR, which included the induction of the molecular chaperone, Bip, CD147 is required to protect HCC cells from ERS-induced apoptosis in vitro and in vivo. Bip forms complexes with the ER membrane-associated caspase-7 and caspase-12 (human caspase-4 is an ortholog of murine caspase-12). Bip overexpression promotes the formation of the Bip-caspase complex and blocks the cleavage of procaspase into its active form, which results in decreased apoptosis. ${ }^{24}$

ADR and VP-16 are widely used as anti-cancer drugs, and they exert their effects by binding to topoisomerase II-DNA complexes, thereby preventing DNA religation after replication. ${ }^{33}$ However, intrinsic and acquired chemoresistance remains an obstacle to the successful management of cancer. UPR activation may diminish topoisomerase II levels, thus conferring cellular resistance to the clinically useful topoisomerase II (Topo II)-directed agents, such as ADR and VP-16. Most excitingly, because our results show that lowering CD147 levels significantly increased the chemosensitivity of cells to ADR and VP-16 and prolonged the survival of nude mice bearing established orthotopic hepatomas. The inhibition of CD147 may therefore represent a novel approach to sensitize HCC to chemotherapy. In this regard, drugs that target CD147 should be combined with existing chemotherapy drugs to increase the effectiveness of chemotherapy and eliminate residual HCC cells after surgery. 
The contribution of Bip to drug resistance has been established for many years, but the lower efficacy of small molecules that downregulate Bip and the inhibition of Bip activity are major challenges to its clinical application. The challenges are due to the fact that, as an ER chaperone protein, Bip resides mainly in the ER lumen. As a transmembrane protein that is expressed on the cell surface, the development of new therapeutic approaches, including small molecules or antibodies that can specifically block CD147 expression and/or its activity, is clinically feasible in the near future. LICARTIN, the ${ }^{131}$ I-labeled Metuximab CD147 antibody (approved as a new drug for clinical therapy of primary HCC patients by the China State Food and Drug Administration (no. S20050039) in April 2005), has already been proven to be safe and effective for the treatment of primary HCC patients and can effectively prevent the recurrence of tumors and prolong the survival of advanced HCC patients after orthotropic liver transplantation. ${ }^{34,35}$ The effects of LICARTIN in overcoming chemoresistance are currently being investigated in our o ongoing investigations.

Thus, we hypothesize that CD147 may not only serve as a diagnostic and prognostic biomarker in HCC, but could also act as a target for promoting apoptosis in HCC cells and increasing their sensitivity to chemotherapy. In addition to its effects in HCC cells, we have also established that CD147 is highly expressed in other cancers, including breast, lung and bladder cancer gliomas and laryngeal squamous cell, ovarian, renal cell and skin carcinomas. ${ }^{20}$ As CD147 is a widely expressed cancer biomarker, its molecular signaling cascade, as well as the mechanism and consequences of its activation in HCC, could also exist in other tumors, as clinical studies and potential translational approaches are utilizing this molecule in developing therapies. The UPR inducer, CD147, provides opportunities to improve the targeting of existing treatments, and its manipulation may either enhance the efficacy of existing agents or represent a novel anti-cancer target in tumor treatments.

\begin{abstract}
Materials and Methods
Cell culture. Human SMMC-7721, HepG2, HEK-293 cells (Institute of Cell Biology, China), 7721-shCD147 and 7721-snc cells (developed in our laboratory) ${ }^{28}$ were cultured in RPMI 1640 medium (Gibco, Grand Island, NE, USA), supplemented with $10 \% \mathrm{FBS}, 1 \%$ penicillin/streptomycin and $2 \%$ L-glutamine at $37^{\circ} \mathrm{C}$ in a humidified atmosphere of $5 \% \mathrm{CO}_{2}$. G418 was added to the medium of the 7721-shCD147 and 7721-snc cell lines at a final concentration of $300 \mu \mathrm{g} / \mathrm{ml}$.
\end{abstract}

Gene silencing. The sense sequences for CD147 siRNAs were $5^{\prime}$-GUUCUU CGUGAGUUCCUCTT-3' (Ambion, Austin, TX, USA). Bip siRNAs were 5'-GGAGC GCAUUGAUACUAGA-3' (Ambion). The cells were transfected with siRNA using the LipofectAMINE 2000 reagent (Invitrogen, Carlsbad, CA, USA) according to the manufacturer's instructions. Silencer negative control siRNA (control siRNA) (Ambion) was used as a negative control under similar conditions.

Immunohistochemistry. A total of 98 cases of human HCC tissues from patients who underwent hepatectomies and 42 normal liver tissues from healthy liver transplant donors (supplied by the Department of Pathology of Xijing Hospital at the Fourth Military Medical University and the Cancer Hospital of Tianjin Medical University) were fixed with $10 \%$ formalin and paraffin embedded. Sections were deparaffinized and incubated with primary antibodies, including mouse CD147 $\mathrm{mAb}$ (prepared in our laboratory) and goat anti-Bip (BD Biosciences, San Jose, CA, USA) antibodies, followed by visualization with a Histostain-Plus Kit (Invitrogen). Immunopositivity was independently evaluated by two pathologists who were blinded to the clinical data. Immunostaining was scored as positive and negative, as described previously. ${ }^{15}$
Western blot analysis. Cells were lysed in $1 \%$ OG buffer $(20 \mathrm{mM} \mathrm{Tris-HCl}$, $\mathrm{pH}$ 8.0, $150 \mathrm{mM} \mathrm{NaCl}, 1 \%$ OG, $1 \mathrm{mM}$ EDTA, $10 \mu \mathrm{g} / \mathrm{ml}$ leupeptin, $2 \mu \mathrm{g} / \mathrm{ml}$ aprotinin and $1 \mathrm{mM}$ PMSF). A BCA Protein Assay Kit (Pierce Biotechnology, Rockford, IL, USA) was then used to determine the total protein density, and equal amounts of protein were separated by $10 \%$ SDS-PAGE and transferred to a polyvinylidene fluoride microporous membrane (Millipore, Billerica, MA, USA). After being blocked with $5 \%$ non-fat milk, the membrane was incubated for $2 \mathrm{~h}$ at room temperature with the designated antibody. A Western-Light chemiluminescent detection system (Applied Biosystems, Foster City, CA, USA) was used for immunodetection.

Real-time quantitative PCR and data analysis. Total RNA was extracted with TRIzol reagent (Invitrogen) and reverse transcribed into cDNA with a ReverTra Ace-a kit (Toyobo, Shanghai, China). All primers and probes were as follows: CD147 forward primer, $5^{\prime}$-TCGCGCTGCTGGGCACC-3', and reverse primer, 5'-TGGCGCTGTCATTCAAGGA-3'; Bip forward primer, 5'-CACAGACGG GTCATTCC-3' ${ }^{\prime}$, and reverse primer, $5^{\prime}$-CCTATGTCGCCTTCACT- $3^{\prime}$; and $\beta$-actin forward primer, $5^{\prime}$-CCCAGCCATGTACGTTGCTA-3', and reverse primer, $5^{\prime}$-TCA CCGGAGT-CCATCACGAT-3'. $\beta$-Actin mRNA was used as an internal control. Real-time quantitative PCR was performed with SYBR green PCR reagent (Takara, Shiga, Japan) and a Stratagene $\mathrm{M} \times$ 3005P Multiplex Quantitative PCR System (Agilent Technologies, Frankfurt am Main, Germany).

Immunofluorescence. After $100 \mathrm{nM} \mathrm{Tg} \mathrm{(Calbiochem,} \mathrm{La} \mathrm{Jolla,} \mathrm{CA,} \mathrm{USA)}$ treatment for $18 \mathrm{~h}$, the cells were allowed to attach to glass coverslips for $6 \mathrm{~h}$. They were then fixed in $3.7 \%$ formaldehyde in PBS, permeabilized with $0.5 \%$ Triton $\mathrm{X}-100$ and blocked with $1 \%$ BSA in PBS for $1 \mathrm{~h}$. Coverslips were then incubated with the indicated antibodies (BD Biosciences) for $20 \mathrm{~min}$. Cells were washed in PBS, incubated with their corresponding FITC secondary antibodies (Pierce) for $1 \mathrm{~h}$ and stained with DAPI (Vector Labs, Burlingame, CA, USA). Finally, the cells were mounted using glycerol and observed with an FV1000 laser scanning confocal microscope (Olympus, Tokyo, Japan).

Nuclear and cytoplasmic protein extraction. Nuclear and cytoplasmic protein extraction was performed using a Nuclear and Cytoplasmic Protein Extraction Kit (Beyotime, Shanghai, China). Briefly, $2 \times 10^{6}$ cells were resuspended in $200 \mu \mathrm{l}$ of cytoplasmic protein isolation solution A and homogenized on ice. Next, $10 \mu \mathrm{l}$ cytoplasmic protein isolation solution B was added, and the cells were homogenized on ice. The homogenate was centrifuged at $10000 \times \mathrm{g}$ for $5 \mathrm{~min}$ at $4{ }^{\circ} \mathrm{C}$. The resulting supernatant was the cytoplasmic protein fraction. The pellet was resuspended in $50 \mu \mathrm{l}$ nuclear protein isolation solution, homogenized on ice and centrifuged at $10000 \times \mathrm{g}$ for $10 \mathrm{~min}$. The resulting supernatant was the nuclear protein fraction.

Chromatin immunoprecipitation. ChIP assay was performed using the ChIP-IT kit (Millipore) according to the manufacturer's protocol. Briefly, the cells were exposed to $100 \mathrm{nM} \mathrm{Tg}$ for $18 \mathrm{~h}$. Cell lysates were incubated with no antibody, immunoglobulin G (IgG), or anti-TFII-I antibody (Abcam, Cambridge, UK). The immunoprecipitated DNA was amplified by the Bip (NC_000009.11) promoterspecific primers. The experimental PCR reactions generated a 160-bp product from the regulatory region of the Bip promoter containing three ERSEs $(-174)$ -15), whereas the control PCR reactions generated a 160-bp product from a distal region without any ERSEs $(+8 /+167)$. Bip ChIP primer: forward $5^{\prime}-G C G A$ AGCTTAGCCGCCGCCGGTCGA-3' reverse 5'-CGAGTAGGCGACGGTGAGG T-3'. Bip control primer: forward $5^{\prime}$-TCTGTCTAACTGGATAACCCCACAA-3' reverse $5^{\prime}$-TGCTCCTGTGCTACGGC-3'.

Plasmid construction. Full-length TFII-I (WT) was PCR-amplified, and the primers were designed as follows: forward primer, $5^{\prime}$-TTGGATCCCGCCCCCCCG CTTCCCCGCACGCGC-3' (BamHI) and reverse primer, 5'-GCTCTAGAAAACTAT GCCGTCACAG-3' (Xbal). The Tyr248 mutant TFII-I (MT) was PCR-amplified using a QuickChange mutagenesis kit (Stratagene, La Jolla, CA, USA) according to the manufacturer's instructions. The tyrosine codon at amino acid 248 in TFII-I cDNA was changed to a phenylalanine codon. The mutation primers used were (mutations are shown in bold): forward primer, $5^{\prime}$-TCAGAAGATCCTGATTTTTATC AATATAACAT-3' and reverse primer, 5'-ATGTTATATTGATAAAAATCAGGATC TTCTGA-3'. The products of full-length TFII-I (WT) and the Tyr248 mutant TFII-I (MT) were confirmed by sequencing (Shanghai Sangon, Shanghai, China) and then cloned into the pcDNA3.1 vector (Promega, Madison, WI, USA), respectively. 
The Bip promoter subregions $(-149 /+7$, which contains all three ERSEs; $-115 /+7$, which contains two ERSEs; $-82 /+7$, which contains just one ERSE; $-49 /+7$, which contains only the minimal TATA promoter; and $-82 /-50$, which contains the first ESRE) were synthesized by Wolsen Biological Technology $\mathrm{Co}_{0}$ and then cloned into the pGL3-Basic vector (Promega). The three BiP promoter ERSEs were disrupted by mutating their respective sequences using a QuickChange mutagenesis kit (Stratagene) as recommended by the manufacturer. The Bip mutation primers used were (mutations are shown in bold): for the first ESRE mutation, forward primer, 5'-AATCGGCGGCGGAATTATTGGTGGC-3' and reverse primer $5^{\prime}$-TTAGCCGCCGCCTTAATAACCACCG-3'; for the second ESRE mutation, forward primer, 5'-AATGAACGGCCTAATTATAGCAGG-3' and reverse primer $5^{\prime}$-TTACTTGCCGGATTAATATCGTCC-3'; and for the third ESRE mutation, forward primer, $5^{\prime}$-TCGGCGGCCTAATTAACGGGGCTGG-3' and reverse primer $5^{\prime}$-AGCCGCCGGATTAATTGCCCCGACC- $3^{\prime}$. The products were cloned into the pGL3-Basic vector respectively.

Transfection experiment. The CD147 expression vector (CD147pcDNA3.1) was developed in our laboratory. ${ }^{36}$ After cells were grown to 60-80\% confluency, transfection was performed using the LipofectAMINE 2000 reagent (Invitrogen) according to the manufacturer's instructions. The pcDNA3.1 empty vector was used as a negative control under similar conditions.

Reporter gene assays. For the luciferase assay, cells were grown to $60-80 \%$ confluence. Bip promoter-reporter gene constructs were then co-transfected with the following vectors using Lipofectamine 2000 (Invitrogen) as recommended by the manufacturer: CD147-pcDNA3.1, TFII-I (WT)-pcDNA3.1 and TFII-I (MT)-pcDNA3.1. The amount of total DNA in each transfection was normalized by the addition of an empty vector to the reaction mixture. For stress induction, the cells were treated with $100 \mathrm{nM} \mathrm{Tg}$ for $18 \mathrm{~h}$ after transfection. The cells were then prepared for the luciferase assays using the Dual-Luciferase Reporter Assay System (Promega). The relative activity was defined as the ratio of firefly luciferase activity to renilla luciferase activity.

Annexin V/PI double staining. The number of dead cells was determined by Annexin V/ PI double staining. Cells were exposed to $100 \mathrm{nM} \mathrm{Tg}$ for $18 \mathrm{~h}$ and then incubated with FITC-conjugated Annexin $\mathrm{V}$ in binding buffer (0.01 M HEPES, $0.14 \mathrm{M} \mathrm{NaCl}$ and $2.5 \mathrm{mM} \mathrm{CaCl}_{2}, \mathrm{pH} 7.4$ ) for $20 \mathrm{~min}$ at $37^{\circ} \mathrm{C}$ in the dark. After incubation, the cells were washed and resuspended in $200 \mu \mathrm{l}$ PBS with $1 \%$ FCS and additionally incubated with $10 \mu \mathrm{l}$ of $1 \mathrm{mg} / \mathrm{ml} \mathrm{PI}$ solution. The Annexin V-positive cells were detected using a FACSCalibur flow cytometer (BD Biosciences), and the results were analyzed using CellQuest software (BD Biosciences). Annexin V-FITC conjugates were detected with the FL1 channel of the FACSCalibur machine. PI was read on the FL2 channel.

Detection of caspase-3 activity. Caspase-3 activity was measured spectrophotometrically via the detection of pNA cleavage from caspase-3-specific substrates. These experiments were completed using a caspase-3 assay kit (Beyotime). After cell lysates were incubated with Ac-LEVD-pNA for $2 \mathrm{~h}$ at $37^{\circ} \mathrm{C}$, the samples were read at $405 \mathrm{~nm}$.

Colony formation assays. Twelve hours after incubation with $100 \mathrm{nM} \mathrm{Tg}$, the cells were seeded in six-well plates. The next day, the medium was changed to include $0.02-2 \mu \mathrm{g} / \mathrm{ml}$ ADR (Hisun Pharma. Co., Zhejiang, China) or $0.2-20 \mu \mathrm{g} / \mathrm{ml}$ Etoposide (VP-16, Qilu, Jinan, China). After a change to fresh medium $48 \mathrm{~h}$ later, the cells were allowed to form colonies for 10 days in the absence of drugs. The colonies were then stained with $0.2 \%$ crystal violet (in $75 \%$ ethanol) for $10 \mathrm{~min}$ and counted.

Hepatoma stably expressing GFP. The cells were infected with a reconstructed pWPT-GFP-puro lentiviral vector, which contains a puromycin selection marker as previously reported. ${ }^{37}$ For selection, $2 \mu \mathrm{g} / \mathrm{ml}$ puromycin was added to the medium, and after 14 days, the medium was changed to medium without puromycin. In this manner, the cells stably expressing GFP were established.

Establishment of an orthotopic HCC model in nude mice. For each mouse, $2 \times 10^{6}$ cells were resuspended in $40 \mu \mathrm{l}$ serum-free DMEM/matrigel. Then, each nude mouse (six in each group, BALB/c-nu/nu) was orthotopically inoculated under anesthesia in the left hepatic lobe with a microsyringe through an 8-mm transverse incision in the upper abdomen. After 25 days, the mice were killed, and their livers were dissected, fixed with phosphate-buffered neutral formalin and prepared for standard histological examination. Animal studies were approved by the local regulatory agency (Laboratory Animal Research Centre of the Fourth Military Medical University).

TUNEL staining. Apoptosis in human tumor xenografts from nude mice sections was detected using the DeadEnd Colorimetric Apoptosis TUNEL System (Promega), which end-labels the DNA from the fragmented apoptotic cells using a modified TUNEL staining according to the manufacturer's instructions.

In-vivo image assay. As described above, $2 \times 10^{6}$ cells stably expressing GFP were implanted in the left hepatic lobe with a microsyringe. The day after implantation, the cells inoculated nude mice were randomized for i.p. treatment with $2.5 \mathrm{mg} / \mathrm{kg}$ ADR (three times, 7 days) or for i.p. treatment with a similar volume of $0.9 \%$ negative control $\mathrm{NaCl}$ solution (three times, 7 days) or were untreated (blank control). At specific time points, GFP imaging was performed using the Xenogen in vivo Imaging System (IVIS 200, Xenogen, Alameda, CA, USA). GFP images were analyzed with Igor image analysis software (Wavemetrics, Lake Oswego, OR, USA). The regions of interest were drawn over the signals, and the GFP images were quantified in units of maximum photons per second per centimeter squared per steradian $\left(\mathrm{p} / \mathrm{s} / \mathrm{cm}^{2} / \mathrm{sr}\right)$. The survival time was also measured for each model.

\section{Conflict of Interest}

The authors declare no conflict of interest.

Acknowledgements. This work was supported by grants from the National Basic Research Program of China (2009CB521704), the National Natural Science Foundation of China $(31101005,81530058,81071691)$ and the National S\&T Major Project (2011ZX09102-001-21, 2012ZX10002017-002).

1. Ashwani $\mathrm{K}$, Arun JS. Endoplasmic reticulum stress and the unfolded protein response. Clin Liver Dis 2009; 13: 581-590.

2. Feldman DE, Chauhan V, Koong AC. The unfolded protein response: a novel component of the hypoxic stress response in tumors. Mol Cancer Res 2005; 3: 597-605.

3. Moenner M, Pluquet $\mathrm{O}$, Bouchecareilh M, Chevet E. Integrated endoplasmic reticulum stress responses in cancer. Cancer Res 2007; 67: 10631-10634.

4. Ron D, Walter P. Signal integration in the endoplasmic reticulum unfolded protein response. Nat Rev Mol Cell Biol 2007; 8: 519-529.

5. Ranganathan AC, Ojha S, Kourtidis A, Conklin DS, Aguirre-Ghiso JA. Dual function of pancreatic endoplasmic reticulum kinase in tumor cell growth arrest and survival. Cancer Res 2008; 68: 3260-3268.

6. Hotamisligil GS. Loss of the tuberous sclerosis complex tumor suppressors triggers the unfolded protein response to regulate insulin signaling and apoptosis. Mol Cell 2008; 29: 541-551

7. Constantinos K, Bradly GW. 'Translating' tumor hypoxia: unfolded protein response (UPR)-dependent and upr-independent pathways. Mol Cancer Res 2006; 4: 423-436.

8. Tsutsumi S, Gotoh T, Tomisato W, Mima S, Hoshino T, Hwang HJ et al. Endoplasmic reticulum stress response is involved in nonsteroidal anti-inflammatory drug-induced apoptosis. Cell Death Differ 2004; 11: 1009-1016.

9. Jianze L, Amy SL. Stress induction of GRP78/BiP and its role in cancer. Curr Mol Med 2006; 6: 45-54.

10. Fernandez PM, Tabbara SO, Jacobs LK, Manning FC, Tsangaris TN, Schwartz AM et al. Overexpression of the glucose-regulated stress gene GRP78 in malignant but not benign human breast lesions. Breast Cancer Res Treat 2000; 59: 15-26.

11. Haynes CM, Titus EA, Cooper AA. Degradation of misfolded proteins prevents ER-derived oxidative stress and cell death. Mol Cell 2004; 15: 767-776.

12. Kondo S, Murakami T, Tatsumi K, Ogata M, Kanemoto S, Otori $\mathrm{K}$ et al. OASIS, a CREB/ATF-family member, modulates UPR signalling in astrocytes. Nat Cell Biol 2005; 7: $186-194$

13. Kouroku Y, Fujita E, Tanida I, Ueno T, Isoai A, Kumagai $H$ et al. ER stress (PERK/elF2alpha phosphorylation) mediates the polyglutamine-induced LC3 conversion, an essential step for autophagy formation. Cell Death Differ 2007; 14: 230-239.

14. Lin JH, Li H, Yasumura D, Cohen HR, Zhang C, Panning B et al. IRE1 signaling affects cell fate during the unfolded protein response. Science 2007; 318: 944-949.

15. Liu FF, Cui LF, Zhang Y, Chen L, Wang YH, Fan Y et al. Expression of HAb18G is associated with tumor progression and prognosis of breast carcinoma. Breast Cancer Res Treat 2010; 124: 677-688. 
16. Ménoret A, Meflah K, Le JP. Expression of the $100 \mathrm{KD}$ glucose regulated protein (GRP100/ endoplasmin) is associated with tumourigenicity in a model rat colon adenocarcinomas Int J Cancer 1994; 56: 400-440.

17. Shuda M, Kondoh N, Imazeki N, Tanaka K, Okada T, Mori K et al. Activation of the ATF6, XBP1 and grp78 genes in human hepatocellular carcinoma: a possible involvement of the ER stress pathway in hepatocarcinogenesis. J Hepatol 2003; 38: 605-614.

18. Cheriyath V, Desgranges ZP, Roy AL. C-Src-dependent transcriptional activation of TFII-I. J Biol Chem 2002; 277: 22798-22805.

19. Ramsay RG, Ciznadija D, Mantamadiotis T, Anderson R, Pearson R. Expression of stress response protein glucose regulated protein-78 mediated by c-Myb. Int J Bioche Cell Biol 2005; 37: 1254-1268.

20. Li Y, Xu J, Chen L, Zhong WD, Zhang Z, Mi L et al. HAb18G (CD147), a cancer-associated biomarker and its role in cancer detection. Histopathology 2009; 54: 677-687.

21. Jiang JL, Yao XY, Zhou J, Huang Y, Chen ZN. Effect of $\mathrm{Ca}^{2+}$ mobilization on release and activation of matrix metalloproteinases in hepatocellular carcinoma cells. Chin $\mathrm{J}$ Oncol 2004; 26: 9525-9527.

22. Tang J, Wu YM, Zhao P, Yang XM, Jiang JL, Chen ZN. Overexpression of CD147 promotes invasion and metastasis via alpha3beta1 integrin mediated FAK-paxillin and FAK-PI3K-Ca ${ }^{2+}$ pathways. Cell Mol Life Sci 2008; 65: 2933-2942.

23. Sammels E, Parys JB, Missiaen L, De SH, Bultynck G. Intracellular $\mathrm{Ca}^{2+}$ storage in health and disease: a dynamic equilibrium. Cell Calcium 2010; 47: 297-314.

24. Fu Y, Li JZ, Amy SL. GRP78/BiP inhibits endoplasmic reticulum BIK and protects human breast cancer cells against estrogen starvation-induced apoptosis. Cancer Res 2007; 67: 3734-3740.

25. Peter S, Nicola J, Brown A, Graham P, Lynda W. The unfolded protein response and cancer: a brighter future unfolding? J Mol Med 2007; 85: 331-341.

26. Parker R, Phan T, Baumeister P, Roy B, Cheriyath V, Roy AL et al. Identification of TFII-I as the endoplasmic reticulum stress response element binding factor ERSF: its autoregulation by stress and interaction with ATF6. Mol Cell Biol 2001; 21: 3220-3233.

27. Novina CD, Cheriyath V, Roy AL. Regulation of TFII-I activity by phosphorylation J Biol Chem 1998; 273: 33443-33448.
28. Li Y, Wu J, Song F, Tang J, Yu XL, Chen ZN, Jiang JL et al. The extracellular membrane-proximal domain of HAb18G/CD147 binds to the MIDAS motif of intrgrin $\beta 1$ to modulate the malignant properties of hepatoma cells. J Biol Chem 2012; 287: 4759-4772.

29. Sato N, Urano F, Yoon LJ, Kim SH, Li M, Donoviel D et al. Upregulation of BiP and CHOP by the unfolded-protein response is independent of presenilin expression. Nat Cell Biol 2000; 2: 863-870.

30. Roy AL, Meisterernst M, Pognonec P, Roeder RG. Cooperative interaction of an initiatorbinding transcription initiation factor and the helix-loop-helix activator USF. Nature 1991; 354: 245-248.

31. Grueneberg DA, Henry RW, Brauer A, Novina CD, Cheriyath V, Roy AL et al. A multifunctional DNA-binding protein that promotes the formation of serum response factor/homeodomain complexes: identity to TFII-I. Genes Dev 1997; 11 2482-2493.

32. Yoshida H, Haze K, Yanagi H, Yura T, Mori K. Identification of the cis-acting endoplasmic reticulum stress response element responsible for transcriptional induction of mammalian glucose-regulated proteins. Involvement of basic leucine zipper transcription factors. J Biol Chem 1998; 273: 33741-33749.

33. Gosky D, Chatterjee S. Down-regulation of topoisomerase II alpha is caused by up-regulation of GRP78. Biochem Biophys Res Commun 2003; 300: 327-332.

34. Chen ZN, Mi L, Xu J, Song F, Zhang Q, Zhang Z et al. Targeting radioimmunotherapy of hepatocellular carcinoma with iodine (131I) Metuximab injection: clinical phase I/II trials. Int $J$ Radiat Oncol Biol Phys 2006; 65: 435-444.

35. Xu J, Shen ZY, Chen XG, Zhang Q, Bian HJ, Zhu P et al. A randomized controlled trial of Licartin for preventing hepatoma recurrence after liver transplantation. Hepatology 2007; 45: 269-276.

36. Jiang JL, Chan HC, Zhou Q, Yu MK, Yao XY, Lam SY et al. CD147-mediated calcium mobilization and hepatoma metastasis require both $\mathrm{C}$-terminal and $\mathrm{N}$-terminal domains. Cell Mol Life Sci 2004; 61: 2083-2091.

37. Moffat J, Grueneberg DA, Yang X, Kim SY, Kloepfer AM, Hinkle G et al. A lentiviral RNAi library for human and mouse genes applied to an arrayed viral high-content screen. Cell 2006; 124: 1283-1298.

Supplementary Information accompanies the paper on Cell Death and Differentiation website (http://www.nature.com/cdd) 